\title{
On the Jäger-Kaul theorem concerning harmonic maps
}

by

\author{
Min-Chun HONG ${ }^{1}$ \\ School of Mathematical Sciences, The Australian National University, \\ Canberra ACT 0200, Australia
}

Manuscript received 30 March 1998

ABSTRACT. - In 1983, Jäger and Kaul proved that the equator map $u^{*}(x)=\left(\frac{x}{|x|}, 0\right): B^{n} \rightarrow S^{n}$ is unstable for $3 \leqslant n \leqslant 6$ and a minimizer for the energy functional $E\left(u, B^{n}\right)=\int_{B^{n}}|\nabla u|^{2} \mathrm{~d} x$ in the class $H^{1,2}\left(B^{n}, S^{n}\right)$ with $u=u^{*}$ on $\partial B^{n}$ when $n \geqslant 7$. In this paper, we give a new and elementary proof of this Jäger-Kaul result. We also generalize the JägerKaul result to the case of $p$-harmonic maps.

(C) 2000 L'Association Publications de l'Institut Henri Poincaré. Published by Elsevier B.V. All rights reserved

RÉSUMÉ. - En 1983, Jäger et Kaul ont demontrés que l'application équateurielle $u^{*}(x)=\left(\frac{x}{|x|}, 0\right): B^{n} \rightarrow S^{n}$ n'est pas stable si $3 \leqslant n \leqslant 6$ et que c'est une minimisateur pour la fonctionelle d'energie $E\left(u, B^{n}\right)=$ $\int_{B^{n}}|\nabla u|^{2} \mathrm{~d} x$ dans la classe $H^{1,2}\left(B^{n}, S^{n}\right)$ avec $u=u^{*}$ sur $\partial B^{n}$ si $n \geqslant 7$. Nous donnons une preuve nouvelle, élémentaire de ce résultat de JägerKaul. En plus nous généralisons le résultat de Jäger-Kaul au cas des applications $p$-harmoniques.

(C) 2000 L'Association Publications de l'Institut Henri Poincaré. Published by Elsevier B.V. All rights reserved

\footnotetext{
${ }^{1}$ Research of the author was supported by the Australian Research Council.
} 


\section{INTRODUCTION}

Let $(M, g)$ be a compact Riemannian manifold with (possibly empty) boundary $\partial M$ and let $(N, h)$ be another compact Riemannian manifold without boundary. Let $u$ be a map from $M$ to $N$ which belongs to $H^{1,2}(M, N)$. We define the energy of $u$ by

$$
E(u, M)=\int_{M}|\mathrm{~d} u|^{2} \mathrm{~d} M,
$$

where $|\mathrm{d} u|$ denotes the Hilbert-Schmidt norm of the differential $\mathrm{d} u(x)$. The critical point of $E$ is called "harmonic". In a fundamental paper [4], Eells and Sampson established existence of smoothly harmonic maps from $M$ to $N$ assuming $N$ has nonpositive section curvature. Let $K \geqslant 0$ be an upper bound for the section curvature of $N$ and $B_{\rho}(q)$ the open geodesic ball in $N$ with center $q$ and radius $\rho$. Assuming essentially the size restriction

$$
u(\partial M) \subset B_{\rho}(q), \quad \rho \leqslant \frac{\pi}{2 \sqrt{K}},
$$

Hildebrandt et al. [10] showed existence of "small" smooth harmonic maps satisfying the condition (1.2) and also discovered that for $n \geqslant 3$ the equator map $u^{*}=\left(\frac{x}{|x|}, 0\right): B^{n} \rightarrow S^{n}$ is a weakly harmonic maps. The uniqueness of harmonic maps in [10] was later proved by Jäger and Kaul [11]. Many important contributions on the regularity of minimizing harmonic maps have been made since then. Schoen and Uhlenbeck [15, 16] obtained that the minimizer of $E$ in $H^{1,2}(M, N)$ is smooth except for a singular set, where the Hausdorff dimension of the singular set is less than or equal to $n-3$. Meanwhile, Giaquinta and Giusti in $[5,6]$ also proved this result for the case when the image lies in a coordinate chart (boundary regularity by Jost and Meier [13]). We would also like to mention Simon's deep works on the structure of singularity of minimizing harmonic maps (e.g., [18]).

Let $B^{n}$ be the unit ball in $\mathbb{R}^{n}$ with boundary $\partial B^{n}=S^{n-1}$, where $S^{n-1}$ is the unit sphere in $\mathbb{R}^{n}$.

A map $u: B^{n} \rightarrow S^{n}$ is called "weakly harmonic" if $u \in H^{1,2}\left(B^{n}, S^{n}\right)$ and it is a critical point of the energy $\int_{B^{n}}|\nabla u|^{2} \mathrm{~d} x$, i.e.,

$$
\int_{B^{n}} \nabla u \cdot \nabla \phi \mathrm{d} x=\int_{B^{n}}|\nabla u|^{2} u \cdot \phi \mathrm{d} x
$$


for all $\phi \in H_{0}^{1,2}\left(B^{n}, \mathbb{R}^{n+1}\right) \cap L^{\infty}\left(B^{n}, \mathbb{R}^{n+1}\right)$.

In 1983, Jäger and Kaul [12] proved the following result:

THEOREM (Jäger-Kaul). -

(i) When $3 \leqslant n \leqslant 6$, the equator map $u^{*}=\left(\frac{x}{|x|}, 0\right)$ is unstable.

(ii) When $n \geqslant 7$, the equator map $u^{*}$ is a minimizer of the energy functional $E\left(u, B^{n}\right)=\int_{B^{n}}|\nabla u|^{2}$ for all maps $u \in H^{1,2}\left(B^{n}, S^{n}\right)$ with $u=u^{*}$ on $\partial B^{n}$.

After this theorem, Giaquinta and Soucek [7] and Schoen and Uhlenbeck [17] proved that the Hausdorff dimension of the singular set of minimizing harmonic maps into a hemisphere is less than or equal to $n-7$.

For any $p \in \mathbb{R}$ with $n>p \geqslant 2$, we define the $p$-energy of maps in $H^{1, p}\left(B^{n}, S^{n}\right)$ by

$$
E_{p}\left(u, B^{n}\right)=\int_{B^{n}}|\nabla u|^{p} \mathrm{~d} x .
$$

A map $u \in H^{1, p}\left(B^{n}, S^{n}\right)$ is called "weakly $p$-harmonic" if $u$ satisfies

$$
\int_{B^{n}}|\nabla u|^{p-2} \nabla u \cdot \nabla \phi \mathrm{d} x=\int_{B^{n}}|\nabla u|^{p} u \cdot \phi \mathrm{d} x
$$

for all $\phi \in H_{0}^{1, p}\left(B^{n}, \mathbb{R}^{n+1}\right) \cap L^{\infty}\left(B^{n}, \mathbb{R}^{n+1}\right)$. It is also easy to check that the equator map $u^{*}$ is a weakly $p$-harmonic map from $B^{n}$ to $S^{n}$ for $2 \leqslant p<n$.

In this paper, we first generalize the Jäger-Kaul theorem to the $p$ energy by the following:

Theorem A. - Assume that $n>p \geqslant 2$.

(i) For $3 \leqslant n<2+p+2 \sqrt{p}$, the equator map $u^{*}=\left(\frac{x}{|x|}, 0\right)$ is an unstable p-harmonic map from $B^{n}$ to $S^{n}$.

(ii) When $n \geqslant 2+p+2 \sqrt{p}$, then $u^{*}$ is a minimizer of the p-energy $E_{p}$ in the class $H^{1, p}\left(B^{n}, S^{n}\right)$ with boundary value $u^{*}$ on $\partial B^{n}$. Moreover, if $n \geqslant 2+p+2 \sqrt{p}, u^{*}$ is the unique minimizer.

We present a new proof of Theorem A(ii) and also point out that our proof is different from and much simpler than the orginal proof by Jäger and Kaul.

Remark a. - When $p=2, n \geqslant 7>4+2 \sqrt{2}$. Thus the proof of Theorem A also extends the Helein theorem in [9, Theorem 3.1] for $n \geqslant 9$ 
to the case when $n \geqslant 7$, i.e., for $n \geqslant 7$, we have

$$
E(u)-E\left(u^{*}\right) \geqslant K_{n}\left\|u-u^{*}\right\|_{H^{1,2}\left(B^{n}\right)}^{2},
$$

where $u$ is a map in $H^{1,2}\left(B^{n}, S^{n}\right)$ which agree with $u^{*}$ on $\partial B^{n}$, and $K_{n}$ is a strict positive constant.

Remark b. - Consider the case of maps from $B^{n}$ into $S^{n-1}$. Then $u^{*}=\frac{x}{|x|}$ is a minimizer of $E\left(u ; B^{n}\right)$ for maps from $B^{n}$ into $S^{n-1}$. This result has been proved by Brezis et al. [2] for $n=3$, by Jäger and Kaul [12] for $n \geqslant 7$ and by Lin [14] for $n \geqslant 3$. Moreover, Coron and Gulliver proved that $u^{*}=\frac{x}{|x|}$ is a minimizer of the $p$-energy functional $E_{p}\left(u ; B^{n}\right)$ for maps from $B^{n}$ into $S^{n-1}$ for $p \leqslant n-1$. Theorem A recovers the partial result of Coron and Gulliver [3] for $n \geqslant 2+p+2 \sqrt{p}$. Perhaps, the uniqueness of the minimizer of $p$-energy functional of maps from $B^{n}$ into $S^{n-1}$ for $n \geqslant 2+p+2 \sqrt{p}$ is also a new result.

Let $M=B^{n}$ again and let $N$ be an ellipsoid of $\mathbb{R}^{n+1}$, i.e.,

$$
N:=\left\{u=(v, z):|v|^{2}+\frac{z^{2}}{a^{2}}=1\right\} \subset \mathbb{R}^{n+1},
$$

where $v \in \mathbb{R}^{n}, z \in \mathbb{R}$, and $a>0$ is a constant.

Baldes [1] in 1984 and Helein [8] in 1988 generalized the work of Jäger and Kaul when $N$ is an ellipsoid:

THEOREM. -

(i) (Baldes) When $a^{2} \geqslant 1$ and $n \geqslant 7$, the equator map $u^{*}$ is the unique minimizer of the energy functional $E$ in $H^{1,2}\left(B^{n} ; N\right)$ with boundary value $(x, 0)$.

(ii) (Helein) If $0<a<1$ and $a^{2} \geqslant 4(n-1) /(n-2)^{2}$, the equator map $u^{*}$ is the unique minimizer of the energy functional $E$ in $H^{1,2}\left(B^{n} ; N\right)$ with boundary value $(x, 0)$.

We would point out that all proofs of Baldes [1] and Helein [8] are variants of the proof of Jäger and Kaul [12]. After the first version of this paper (CMA-Preprint, September 1996), the author was asked whether one could recover and generalize the results of Baldes and Helein using our proof of Theorem A. Here we generalize their results to $p$-harmonic maps with $n>p \geqslant 2$ by the following: 
THEOREM B. -

(i) When $a^{2} \geqslant 1$ and $n \geqslant 2+p+2 \sqrt{p}$, the equator map $u^{*}$ is the unique minimizer of the p-energy functional $E_{p}$ in $H^{1, p}\left(B^{n} ; N\right)$ with boundary value $(x, 0)$.

(ii) If $0<a<1$ and $a \geqslant 4(n-1) /(n-p)^{2}$, the equator map $u^{*}$ is the unique minimizer of the p-energy functional $E_{p}$ in $H^{1, p}\left(B^{n} ; N\right)$ with boundary value $(x, 0)$.

\section{PROOF OF THEOREM A}

LEMMA 1. - For any $p<n$, we have

$$
(n-p)^{2} \int_{B^{n}}|\phi(x)|^{2} \frac{1}{|x|^{p}} \mathrm{~d} x \leqslant 4 \int_{B^{n}} \frac{1}{|x|^{p-2}}\left|\frac{\partial \phi}{\partial r}\right|^{2} \mathrm{~d} x
$$

for all $\phi \in H_{0}^{1, p} \cap L^{\infty}\left(B^{n}, \mathbb{R}\right)$ with $r=|x|$ where that equality occurs only if $\phi=0$.

Proof. - The case of $p=2$ was proved in [1]. Integrating by parts and using Cauchy's inequlaity, we have

$$
\begin{aligned}
\int_{B^{n}}|\phi|^{2} \frac{1}{|x|^{p}} \mathrm{~d} x & =\int_{|\omega|=1} \int_{0}^{1} \phi^{2} r^{n-p-1} \mathrm{~d} r \mathrm{~d} \omega \\
& =\frac{2}{n-p} \int_{|\omega|=1} \int_{0}^{1} \phi \frac{\partial \phi}{\partial r} r^{n-p} \mathrm{~d} r \mathrm{~d} \omega \\
& \leqslant \frac{1}{2} \int_{|\omega|=1} \int_{0}^{1} \phi^{2} r^{n-p-1} \mathrm{~d} r \mathrm{~d} \omega \\
& +\frac{2}{(n-p)^{2}} \int_{|\omega|=1}^{1}\left(\frac{\partial \phi}{\partial r}\right)^{2} r^{n-p+1} \mathrm{~d} r \mathrm{~d} \omega
\end{aligned}
$$

for all $\phi \in H_{0}^{1, p} \cap L^{\infty}\left(B^{n}, \mathbb{R}\right)$. The above inequality becomes equality iff

$$
\phi=\frac{2}{(n-p)} \frac{\partial \phi}{\partial r}
$$

this is psossible ony if $\phi=0$. This proves our claim. 
Remark c. - Lemma 1 can be also proved in following way:

$$
\begin{gathered}
\inf _{\phi \neq 0, \operatorname{supp} \phi \subset \bar{B}^{n} \backslash\{0\}} \frac{\int_{B^{n}}|\nabla \phi|^{2} r^{-(p-2)} \mathrm{d} x}{\int_{B^{n}} r^{-p}|\phi|^{2} \mathrm{~d} x} \\
=\inf _{\tilde{\phi} \equiv 0, \operatorname{supp} \tilde{\phi} \subset(0,1]} \frac{\int_{0}^{1}\left|\frac{\partial \tilde{\phi}}{\partial r}\right|^{2} r^{n+1-p} \mathrm{~d} r}{\int_{0}^{1}|\tilde{\phi}|^{2} r^{n-1-p} \mathrm{~d} r} \\
\geqslant \inf _{\tilde{\phi} \neq 0, \operatorname{supp} \tilde{\phi} \subset(0, \infty)} \frac{\int_{0}^{\infty}\left|\frac{\partial \tilde{\phi}}{\partial r}\right|^{2} r^{n+1-p} \mathrm{~d} r}{\int_{0}^{\infty}|\tilde{\phi}|^{2} r^{n-1-p} \mathrm{~d} r}=\frac{(n-p)^{2}}{4} .
\end{gathered}
$$

This can be done by modifying a lemma taken from [17, Lemma 1.3].

\subsection{Proof of Theorem A(ii)}

Let $u^{*}=\left(\frac{x}{|x|}, 0\right)$ be the "equator map" from $B^{n} \rightarrow S^{n}$. It is easy to see

$$
\left|\nabla u^{*}\right|^{2}=\frac{n-1}{r^{2}} .
$$

Let $w \in H^{1,2}\left(B^{n}, S^{n}\right)$ be any function with boundary value $w=u^{*}$ on $\partial B^{n}$.

By Lemma 1, we obtain

$$
\int_{B^{n}}\left|\nabla u^{*}\right|^{p-2}|\nabla \phi|^{2} \mathrm{~d} x \geqslant \frac{(n-p)^{2}}{4(n-1)} \int_{B^{n}}\left|\nabla u^{*}\right|^{p} \phi^{2} \mathrm{~d} x .
$$

When $n \geqslant 2+p+\sqrt{4 p}$, we have

$$
\frac{(n-p)^{2}}{4(n-1)} \geqslant 1
$$

When $n \geqslant 2+p+\sqrt{4 p}$, we get

$$
\int_{B^{n}}\left|\nabla u^{*}\right|^{p-2}\left|\nabla\left(u^{*}-w\right)\right|^{2} \mathrm{~d} x \geqslant \int_{B^{n}}\left|\nabla u^{*}\right|^{p}\left(u^{*}-w\right)^{2} \mathrm{~d} x
$$

for all $w \in H^{1, p}\left(B^{n}, S^{n}\right)$ with $w=u^{*}$ on $\partial B^{n}$. Moreover, we know

$$
\int_{B^{n}}\left|\nabla u^{*}\right|^{p-2}\left|\nabla\left(u^{*}-w\right)\right|^{2} \mathrm{~d} x
$$




$$
\begin{aligned}
& =\int_{B^{n}}\left|\nabla u^{*}\right|^{p} \mathrm{~d} x-2 \int_{B^{n}}\left|\nabla u^{*}\right|^{p-2} \nabla u^{*} \cdot \nabla w \mathrm{~d} x \\
& \quad+\int_{B^{n}}\left|\nabla u^{*}\right|^{p-2}|\nabla w|^{2} \mathrm{~d} x
\end{aligned}
$$

and

$$
\int_{B^{n}}\left|\nabla u^{*}\right|^{p}\left(u^{*}-w\right)^{2} \mathrm{~d} x=\int_{B^{n}}\left|\nabla u^{*}\right|^{p}\left(2-2 u^{*} \cdot w\right) \mathrm{d} x .
$$

Notice that $u^{*}$ is a weakly $p$-harmonic map, i.e.,

$$
\int_{B^{n}}\left|\nabla u^{*}\right|^{p-2} \nabla u^{*} \cdot \nabla \phi \mathrm{d} x=\int_{B^{n}}\left|\nabla u^{*}\right|^{p} u^{*} \cdot \phi \mathrm{d} x
$$

for all $\phi \in H_{0}^{1, p}\left(B^{n}, \mathbb{R}^{n+1}\right)$. By taking $\phi=u^{*}-w$, we have

$$
\int_{B^{n}}\left|\nabla u^{*}\right|^{p-2} \nabla u^{*} \cdot \nabla w \mathrm{~d} x=\int_{B^{n}}\left|\nabla u^{*}\right|^{p} u^{*} \cdot w \mathrm{~d} x
$$

From (2.1)-(2.4), we get for $n \geqslant 2+p+\sqrt{4 p}$

$$
\int_{B^{n}}\left|\nabla u^{*}\right|^{p-2}|\nabla w|^{2} \mathrm{~d} x \geqslant \int_{B^{n}}\left|\nabla u^{*}\right|^{p} \mathrm{~d} x
$$

By the Hölder inequaity, we have

$$
\int_{B^{n}}\left|\nabla u^{*}\right|^{p-2}|\nabla w|^{2} \mathrm{~d} x \leqslant\left(\int_{B^{n}}\left|\nabla u^{*}\right|^{p} \mathrm{~d} x\right)^{(p-2) / p}\left(\int_{B^{n}}|\nabla w|^{p} \mathrm{~d} x\right)^{2 / p} .
$$

Combing two above inequalities, we have

$$
\int_{B^{n}}\left|\nabla u^{*}\right|^{p} \mathrm{~d} x \leqslant \int_{B^{n}}|\nabla w|^{p} \mathrm{~d} x
$$

for all $w \in H^{1, p}\left(B^{n}, S^{n}\right)$ with boundary value $w=u^{*}$ on $\partial B^{n}$ when $n \geqslant 2+p+2 \sqrt{p}$. 
From Lemma 1, we know that (2.1) become equality only if $w=u^{*}$. If $n \geqslant 2+p+2 \sqrt{p}$, we have

$$
\int_{B^{n}}\left|\nabla u^{*}\right|^{p} \mathrm{~d} x<\int_{B^{n}}|\nabla w|^{p} \mathrm{~d} x
$$

for all $w \in H^{1, p}\left(B^{n}, S^{n}\right)$ with boundary value $w=u^{*}$ on $\partial B^{n}$ and $w \not \equiv u^{*}$. It means that $u^{*}$ is the unique minimizer for $n \geqslant 2+p+2 \sqrt{p}$. This proves Theorem $\mathrm{A}(\mathrm{ii})$.

Assume that $p=2$. When $n \geqslant 7>4+2 \sqrt{2}$, one sees $(n-2)^{2} /(4$ $(n-1))>1$. By Lemma 1, we know

$$
\int_{B^{n}}|\nabla \phi|^{2} \mathrm{~d} x \geqslant\left(1-\frac{4(n-1)}{(n-2)^{2}}\right) \int_{B^{n}}|\nabla \phi|^{2} \mathrm{~d} x+\int_{B^{n}}\left|\nabla u^{*}\right|^{2} \phi^{2} \mathrm{~d} x
$$

for all $\phi \in H_{0}^{1,2}\left(B^{n}, \mathbb{R}^{n+1}\right)$. Taking $\phi=w-u^{*}$, we have

$$
\int_{B^{n}}|\nabla w|^{2} \mathrm{~d} x-\int_{B^{n}}\left|\nabla u^{*}\right|^{2} \mathrm{~d} x \geqslant\left(1-\frac{4(n-1)}{(n-2)^{2}}\right) \int_{B^{n}}\left|\nabla\left(w-u^{*}\right)\right|^{2} \mathrm{~d} x,
$$

where $w \in H^{1,2}\left(B^{n}, S^{n}\right)$ agrees with $u^{*}$ on $\partial B^{n}$. This proves our claim in Remark 1.

For $t$ small we define $u_{t}: B^{n} \rightarrow S^{n}$ by setting

$$
u_{t}(x)=\frac{\left(\frac{x}{|x|}, t \phi(x)\right)}{\left(1+t^{2} \phi^{2}\right)^{1 / 2}}
$$

for a smooth function $\phi$ on $B^{n}$ vanishing near 0 and $\partial B^{n}$. A simple calculation gives

$$
\left.\frac{\partial \nabla u_{t}(x)}{\partial t}\right|_{t=0}=(0, \ldots, 0, \nabla \phi(x)),\left.\quad \frac{\partial}{\partial t}\left|\nabla u_{t}\right|^{2}\right|_{t=0}=0
$$

and

$$
\left.\frac{\partial^{2} \nabla u_{t}(x)}{\partial^{2} t}\right|_{t=0}=\phi^{2}\left(\nabla \frac{x}{|x|}, 0\right)
$$

Now

$$
\left.\frac{1}{2} \frac{\partial^{2}}{\partial^{2} t}\left|\nabla u_{t}\right|^{2}\right|_{t=0}=\left|\frac{\partial \nabla u_{t}(x)}{\partial t}\right|_{t=0}^{2}+\left(\frac{\partial^{2} \nabla u_{t}(x)}{\partial^{2} t}, \nabla u_{t}\right)_{t=0} .
$$


Then

$$
\left.\frac{\mathrm{d}^{2}}{\mathrm{~d}^{2} t} E_{p}\left(u_{t}\right)\right|_{t=0}=\int_{B^{n}}\left|\nabla u^{*}\right|^{p-2}\left[|\nabla \phi|^{2}-\phi^{2}\left|\nabla u^{*}\right|^{2}\right] \mathrm{d} x
$$

It is easy to check that $u^{*}=\left(\frac{x}{|x|}, 0\right)$ is a weakly $p$-harmonic map from $B^{n}$ into $S^{n}$. If $u^{*}$ is stable, we have

$$
\int_{B^{n}}\left|\nabla u^{*}\right|^{p-2}\left[|\nabla \phi|^{2}-\phi^{2}\left|\nabla u^{*}\right|^{2}\right] \mathrm{d} x \geqslant 0
$$

for all smooth $\phi$ vanishing near 0 and $\partial B^{n}$.

\subsection{Proof of Theorem A(i)}

Let us consider the following equation:

$$
\left\{\begin{array}{l}
\phi^{\prime \prime}(r)+\frac{n-p+1}{r} \phi^{\prime}(r)+\frac{n-1-\varepsilon}{r^{2}} \phi(r)=0 \\
\phi\left(r_{0}\right)=\phi(1)=0
\end{array}\right.
$$

for $0<r_{0}<1$.

By setting $\xi(t)=\phi\left(e^{t}\right)$, Eq. (2.5) becomes

$$
\xi^{\prime \prime}(t)+(n-p) \xi^{\prime}(t)+(n-1-\varepsilon) \xi(t)=0 .
$$

Let

$$
v:=v(\varepsilon)=\frac{1}{4}\left[n^{2}-2(p+2) n+p^{2}\right]+1+\varepsilon .
$$

When $3 \leqslant n<2+p+2 \sqrt{p}$, there exists a small $\varepsilon$ such that $v<0$ and we choose $r_{0}: 0<r_{0}<1$ such that $\sqrt{-v} \ln r_{0}$ is a multiple of $2 \pi$. Then it is easily checked (see [12]) that the function

$$
\phi(r)= \begin{cases}r^{(p-n) / 2} \sin (\sqrt{-v} \cdot \ln r), & \text { for } r_{0}<r \leqslant 1, \\ 0, & \text { for } r \leqslant r_{0} .\end{cases}
$$


solves Eq. (2.5). This means that for $3 \leqslant n<2+p+2 \sqrt{p}$, there exists $\varepsilon$ small and a non-zero $\phi(r)$ on $\left[r_{0}, 1\right], \phi(1)=\phi\left(r_{0}\right)=0$, such that

$$
\int_{0}^{1} r^{2-p}\left[\phi^{\prime}(r)^{2}-\frac{(n-1)}{r^{2}} \phi^{2}\right] r^{n-1} \mathrm{~d} r=-\int_{0}^{1} \frac{\varepsilon}{r^{2}} r^{2-p} \phi^{2} r^{n-1} \mathrm{~d} r<0 .
$$

In other words, we see that $u^{*}$ is unstable for $3 \leqslant n<2+p+2 \sqrt{p}$. This proves Theorem A(i).

From the proof of Theorem A, we have

COROLlaRY 2. - Assume that $u$ is a stable p-harmonic map from $B^{n}$ into $S^{n}$ and the values of $u$ are on the equator $\left(S^{n-1}, 0\right)$ of $S^{n}$. Then $u$ is a local minimizer of the energy functional $E_{p}$ in $H^{1, p}\left(B^{n}, S^{n}\right)$.

\section{PROOF OF THEOREM B}

In this section, let $N$ be the ellipsoid of $\mathbb{R}^{n+1}$ defined in Section 1 and suppose that $p \in \mathbb{R}$ with $n>p \geqslant 2$. We define the $p$-energy of maps in $H^{1, p}\left(B^{n}, N\right)$ by

$$
E_{p}\left(v, z ; B^{n}\right)=\int_{B^{n}}\left(|\nabla v|^{2}+|\nabla z|^{2}\right)^{p / 2} \mathrm{~d} x .
$$

We write $u=(v, z)$ with $v \in \mathbb{R}^{n}, z \in \mathbb{R}$. A map $u=(v, z) \in H^{1, p}\left(B^{n}, N\right)$ is called "weakly $p$-harmonic" if $u$ satisfies in the sense of distributions the following equations:

$$
\begin{gathered}
\operatorname{div}\left(|\nabla u|^{p-2} \nabla v\right)+|\nabla u|^{p-2} \lambda v=0, \\
\operatorname{div}\left(|\nabla u|^{p-2} \nabla z\right)+|\nabla u|^{p-2} \lambda \frac{z}{a^{2}}=0,
\end{gathered}
$$

where

$$
\lambda=\left(|\nabla v|^{2}+\frac{|\nabla z|^{2}}{a^{2}}\right) \frac{a^{4}}{a^{4} v^{2}+z^{2}} .
$$

Proof of Theorem B. - Let $u=(v, z)$ be any function in $H^{1, p}\left(B^{n}, N\right)$ with boundary values $(x, 0)$ on $\partial B^{n}$. Using Lemma 1 again, we have

$$
\int_{B^{n}}\left|\nabla \frac{x}{|x|}\right|^{p-2}\left|\nabla\left(\frac{x}{|x|}-v\right)\right|^{2} \mathrm{~d} x
$$




$$
\geqslant \frac{(n-p)^{2}}{4(n-1)} \int_{B^{n}}\left|\nabla \frac{x}{|x|}\right|^{p}\left|\frac{x}{|x|}-v\right|^{2} \mathrm{~d} x
$$

and

$$
\int_{B^{n}}\left|\nabla \frac{x}{|x|}\right|^{p-2}|\nabla z|^{2} \mathrm{~d} x \geqslant \frac{(n-p)^{2} a^{2}}{4(n-1)} \int_{B^{n}}\left|\nabla \frac{x}{|x|}\right|^{p} \frac{|z|^{2}}{a^{2}} \mathrm{~d} x .
$$

(i) Assume that $a \geqslant 1$ and $n \geqslant 2+p+2 \sqrt{p}$. Note the fact $v^{2}+\frac{z^{2}}{a^{2}}=1$. Thus using (3.1) and (3.2), the proof of Theorem A(ii) yields

$$
\int_{B^{n}}\left|\nabla \frac{x}{|x|}\right|^{p-2}|\nabla u|^{2} \mathrm{~d} x \geqslant \frac{(n-p)^{2}}{4(n-1)} \int_{B^{n}}\left|\nabla \frac{x}{|x|}\right|^{p} \mathrm{~d} x
$$

for all $u=(v, z)$ with same boundary values $(x, 0)$. The same argument in the proof of Theorem A(ii) gives that $\left(\frac{x}{|x|}, 0\right)$ is the unique minimizer of $E_{p}$ if $n \geqslant 2+p+2 \sqrt{p}$.

(ii) Assume that $0<a<1$ and $a^{2} \geqslant 4(n-1) /(n-p)^{2}$. Thus using (3.1) and (3.2) again, we get

$$
\int_{B^{n}}\left|\nabla \frac{x}{|x|}\right|^{p-2}|\nabla u|^{2} \mathrm{~d} x>\frac{(n-p)^{2} a^{2}}{4(n-1)} \int_{B^{n}}\left|\nabla \frac{x}{|x|}\right|^{p} \mathrm{~d} x
$$

for all $u=(v, z) \not \equiv\left(\frac{x}{|x|}, 0\right)$ with same boundary values. The same argument in the proof of Theorem $\mathrm{A}(\mathrm{ii})$ gives that $\left(\frac{x}{|x|}, 0\right)$ is the unique minimizer of $E_{p}$. This proves Theorem B.

Remark d. - It is obvious from the proof of Theorem A(i) to see that $\left(\frac{x}{|x|}, 0\right)$ is unstable for $E_{p}$ when $a^{2}<4(n-p) /(n-2)^{2}$.

\section{REFERENCES}

[1] A. BALDES, Stability and uniqueness properties of the equator map from a ball into an ellipsoid, Math. Z. 185 (1984) 505-516.

[2] H. BreZis, J.-M. Coron and E.H. Lieb, Harmonic maps with defects, Comm. Math. Phys. 107 (1986) 649-705.

[3] J.-M. Coron and R. Gulliver, Minimizing $p$-harmonic maps into spheres, J. Reine Angew. Math. 401 (1989) 82-100.

[4] J. EELLS and J.H. SAMPSON, Harmonic mappings of Riemannian manifolds, Amer. J. Math. 86 (1964) 109-160. 
[5] M. Giaquinta and E. Giusti, On the regualrity of the minima of variational integrals, Acta Math. 148 (1982) 31-46.

[6] M. Giaquinta and E. Giusti, The singular set of the minima of certain quadratic functionals, Ann. Scuola Norm. Sup. Pisa (4) 11 (1984) 45-55.

[7] M. Giaquinta and J. SouceK, Harmonic maps into a hemisphere, Ann. Scuola Norm. Sup. Pisa (4) 12 (1985) 81-90.

[8] F. Helein, Regularity and uniqueness of harmonic maps into an ellipsoid, Manuscripta Math. 60 (1988) 235-257.

[9] F. HeLEIN, Approximations of Sobolev maps between an open set and an Euclidean sphere, boundary data and singularities, Math. Ann. 285 (1989) 125-140.

[10] S. Hildebrandt, H. KaUl and K.-O. Widman, An existence theorem for harmonic mappings of Riemannian manifolds, Acta Math. 138 (1977) 1-16.

[11] W. JÄGER and H. KAUL, Uniqueness and stability of harmonic maps and their Jacobi fields, Manuscripta Math. 28 (1979) 269-291.

[12] W. JÄGER and H. KAUL, Rotationally symmetric harmonic maps from a ball into a sphere and the regularity problem for weak solutions of elliptic systems, J. Reine Angew. Math. 343 (1983) 146-161.

[13] J. Jost and M. MEIER, Boundary regularity for minima of certain quadratic functionals, Math. Ann. 262 (1983) 549-561.

[14] F.-H. Lin, Une remarque sur l'application $x /|x|$, C. R. Acad. Sci. Paris 305 (1987) 529-531.

[15] R. SCHOEN and K. UhLENBECK, A regularity theory for harmonic maps, J. Differential Geom. 17 (1982) 307-335.

[16] R. Schoen and K. Uhlenbeck, Boundary theory and the Dirichlet problem for harmonic maps, J. Differential Geom. 18 (1983) 253-268.

[17] R. SCHOEN and K. UHLENBECK, Regularity of minimizing harmonic maps into the sphere, Invent. Math. 78 (1984) 89-100.

[18] L. Simon, Theorems on Regularity and Singularity of Energy Minmizing Maps, Lecture Notes in Mathematics, ETH Zürich, Birkhäuser, Basel, 1996. 